\title{
The Challenging Clinical Management of Patients with Cranial Dural Arteriovenous Fistula and Secondary Parkinson's Syndrome: Pathophysiology and Treatment Options
}

\author{
Julia Velz ${ }^{a, b}$ Zsolt Kulcsar ${ }^{b, c}$ Fabian Büchele ${ }^{b, d}$ Heiko Richter ${ }^{e}$ \\ Luca Reglia, b \\ a Department of Neurosurgery, Clinical Neuroscience Center, University Hospital \\ Zurich, Zurich, Switzerland; b University of Zurich, Zurich, Switzerland; ' ${ }^{\mathrm{D}}$ Department \\ of Neuroradiology, Clinical Neuroscience Center, University Hospital Zurich, Zurich, \\ Switzerland; ${ }^{d}$ Department of Neurology, Clinical Neuroscience Center, University Hospital \\ Zurich, Zurich, Switzerland; e Department of Neurosurgery, Kantonsspital St. Gallen, \\ St. Gallen, Switzerland
}

\section{Keywords}

Intracranial dural arteriovenous fistula · Venous sinus thrombosis · Shunt - Venous hypertension · Parkinsonism

\begin{abstract}
Cranial dural arteriovenous fistula (CDAVF) may rarely lead to parkinsonism and rapid cognitive decline. Dysfunction of the extrapyramidal system and the thalamus, due to venous congestion of the Galenic system with subsequent parenchymal edema, is likely to represent an important pathophysiological mechanism. Here, we report a case of a 57-year-old man with a CDAVF of the straight sinus (Borden type III; DES-Zurich bridging vein shunt [BVS] type with direct, exclusive, and strained leptomeningeal venous drainage [LVD]) and subsequent edema of both thalami, the internal capsule, the hippocampi, the pallidum, and the mesencephalon. Several attempts at venous embolization were unsuccessful, and the neurological condition of the patient further deteriorated with progressive parkinsonism and intermittent episodes of loss of consciousness (KPS 30). A suboccipital mini-craniotomy was performed and the culminal vein was disconnected from the medial tentorial sinus, achieving an immediate fistula occlusion. Three-month follow-up MRI revealed complete regression of the edema. Clinically, parkinsonism remitted completely, allowing for tapering of dopaminergic medication. His cognition markedly improved in further course. The purpose of this report is to highlight the importance of rapid and complete CDAVF occlusion to reverse venous hypertension and pre-
\end{abstract}


vent progressive clinical impairment. The review of the literature underlines the high morbidity and mortality of these patients. Microsurgical disconnection of the fistula plays an important role in the management of these patients and, surprisingly, has not been reported so far.

(C) 2020 The Author(s)

Published by S. Karger AG, Basel

\section{Introduction}

A cranial dural arteriovenous fistula (cDAVF) is an acquired vascular malformation characterized by arteriovenous (AV) shunting within one of the following venous structures: the dural sinus (dural sinus shunt [DSS]), the transdural segment of the bridging veins (bridging vein shunt [BVS]), or the emissary and epidural veins (emissary vein shunt [EVS]) [1-3]. cDAVF represents approximately $10-15 \%$ of all intracranial AV malformations and has been described as being caused by traumatic injuries, venous sinus inflammation, brain surgery, and a hypercoagulable state [4], but the most plausible explanation of its development is sinus thrombosis, mostly as consequence of the previously listed conditions [2,5].

The clinical manifestation of cDAVF depends on its anatomic location and the pattern of venous drainage. cDAVF with leptomeningeal venous drainage (LVD), also called cortical venous reflux, carries the highest risk of an aggressive clinical course and may present with venous congestion-related parenchymal edema, microbleeds, or massive hemorrhages, and subarachnoid bleeding due to the rupture of venous varcies [6]. Borden et al. [4] classified these lesions as type 2 and type 3. Cognard et al. [7] described them as type IIb, IIa + IIb, III, IV, and V. According to the DES (ㅁirect, exclusive, strained)-Zurich classification, LVD is an inherent characteristic of all BVS-type fistulae. Due to their specific venous angioachitecture, DSS-type fistulae and EVS present with LVD frequently and infrequently, respectively $[2,3,6]$.

A rare, but severe consequence of cDAVF at a specific location is the occurrence of parkinsonism with/or without progressive cognitive decline. Parkinsonism as a clinical manifestation of cDAVF is exceptionally rare and has only been reported in case reports (Table 1) [8-26]. Dysregulation of the extrapyramidal system and the thalamus due to venous congestion of the Galenic system, with subsequent parenchymal edema, seems to represent an important pathophysiological mechanism here [20].

The early diagnosis and prompt treatment of cDAVF with LVD is mandatory to reverse the venous congestion and eliminate the risk of bleeding [4]. Complete occlusion of the cDAVF is obligatory to achieve neurological recovery and needs to be urgently performed. However, the incidence of incomplete cDAVF occlusion in these patients, which leads to further neurological deterioration, and even death, is surprisingly high in the literature $[11,12,14,22$, $24,25]$.

The rare incidence of cDAVF leading to parkinsonism and cognitive dysfunction and the challenging treatment prompted us to report this case and discuss the current management of cranial cDAVF based on a literature review.

\section{Case Report}

A 57-year-old man presented to an external hospital due to rapidly progressive cognitive impairment over 3 months. Neurological examination revealed an altered mental status, with impaired executive function and a hypokinetic movement disorder with gait impairment, hypophonia, and hypomimia. The patient had known reflux esophagitis but no other signif-

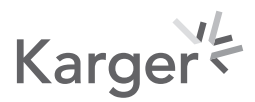


Cerebrovascular Diseases Extra
Cerebrovasc Dis Extra 2020;10:124-138 \begin{tabular}{l|l}
\hline DOI: $10.1159 / 000510597$ & (c) 2020 The Author(s). Published by S. Karger AG, Basel
\end{tabular} www.karger.com/cee

Velz et al.: cDAVF and Secondary Parkinson's Syndrome

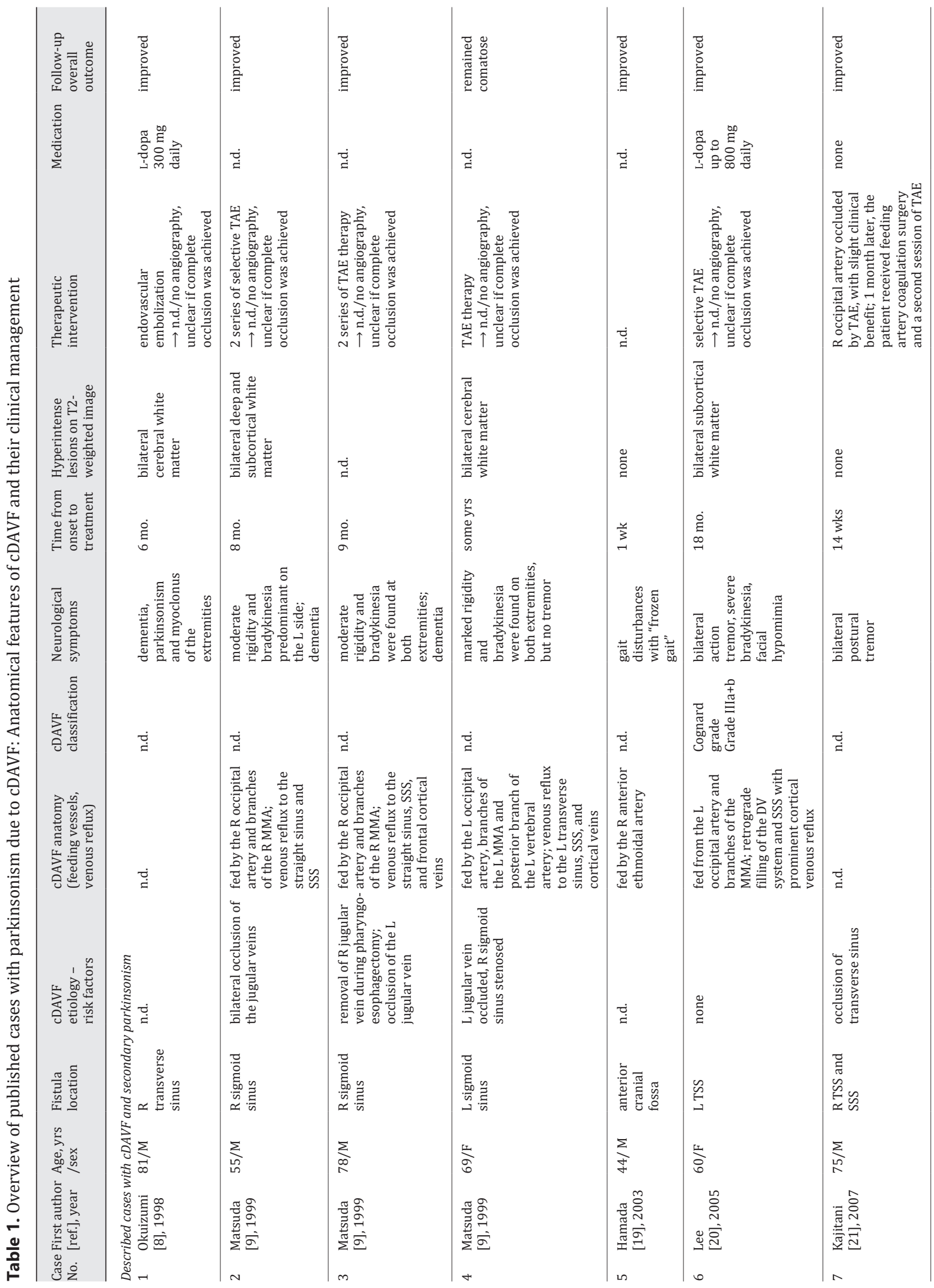


Cerebrovascular Diseases Extra
Cerebrovasc Dis Extra 2020;10:124-138

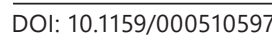

(c) 2020 www.karger.com/cee

Velz et al.: cDAVF and Secondary Parkinson's Syndrome

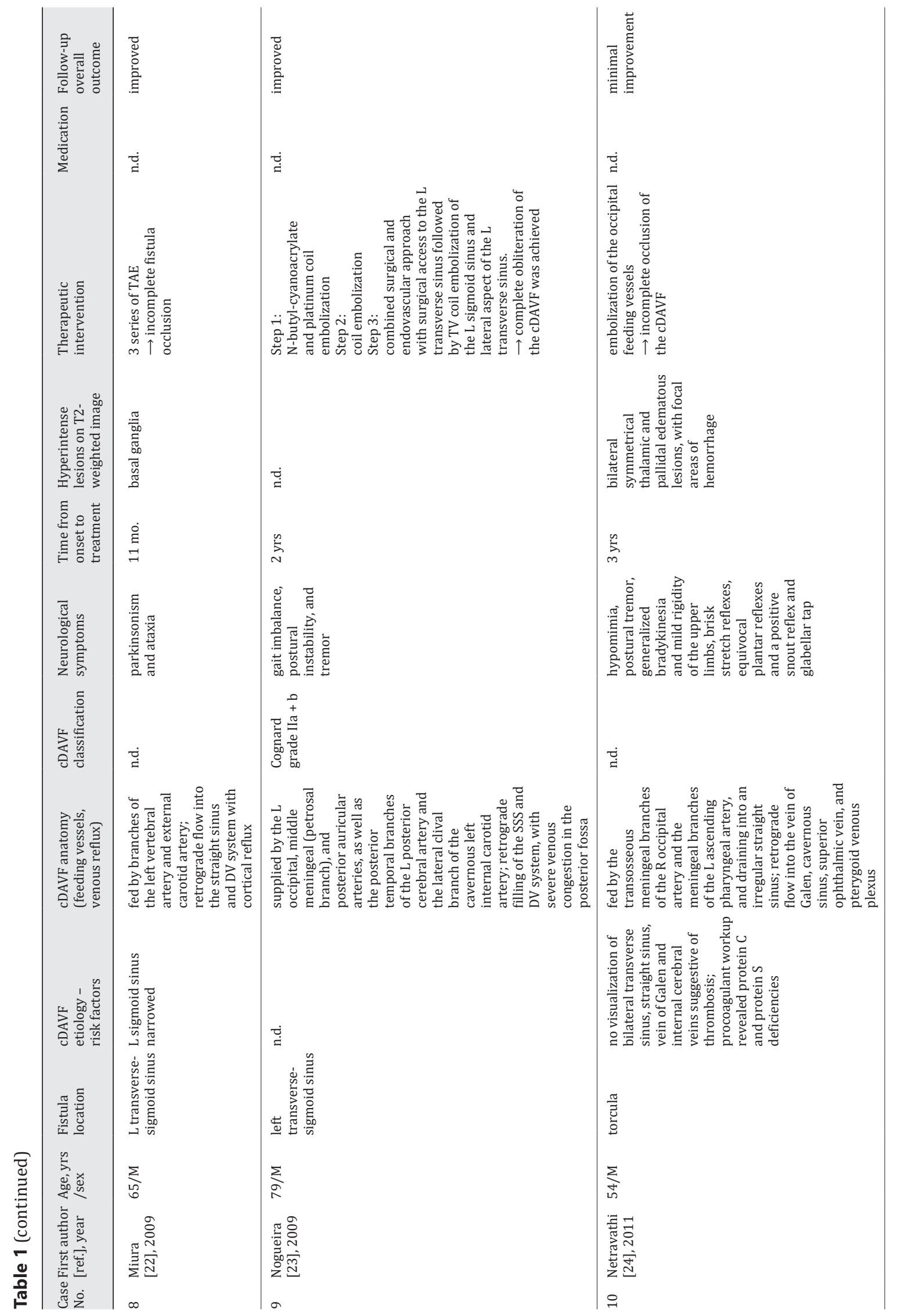

Karger'k 
Cerebrovascular Diseases Extra

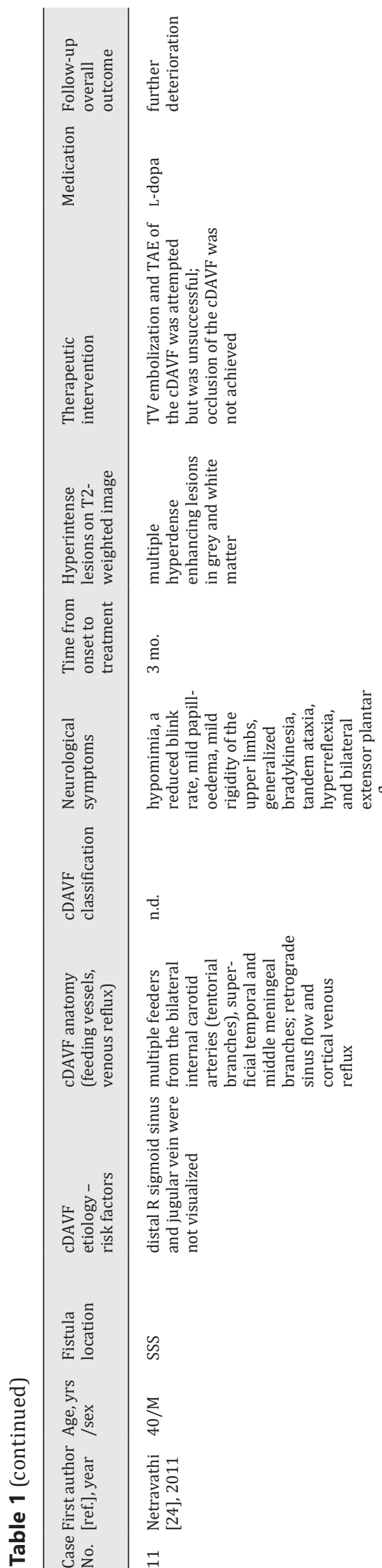

Cerebrovasc Dis Extra 2020;10:124-138

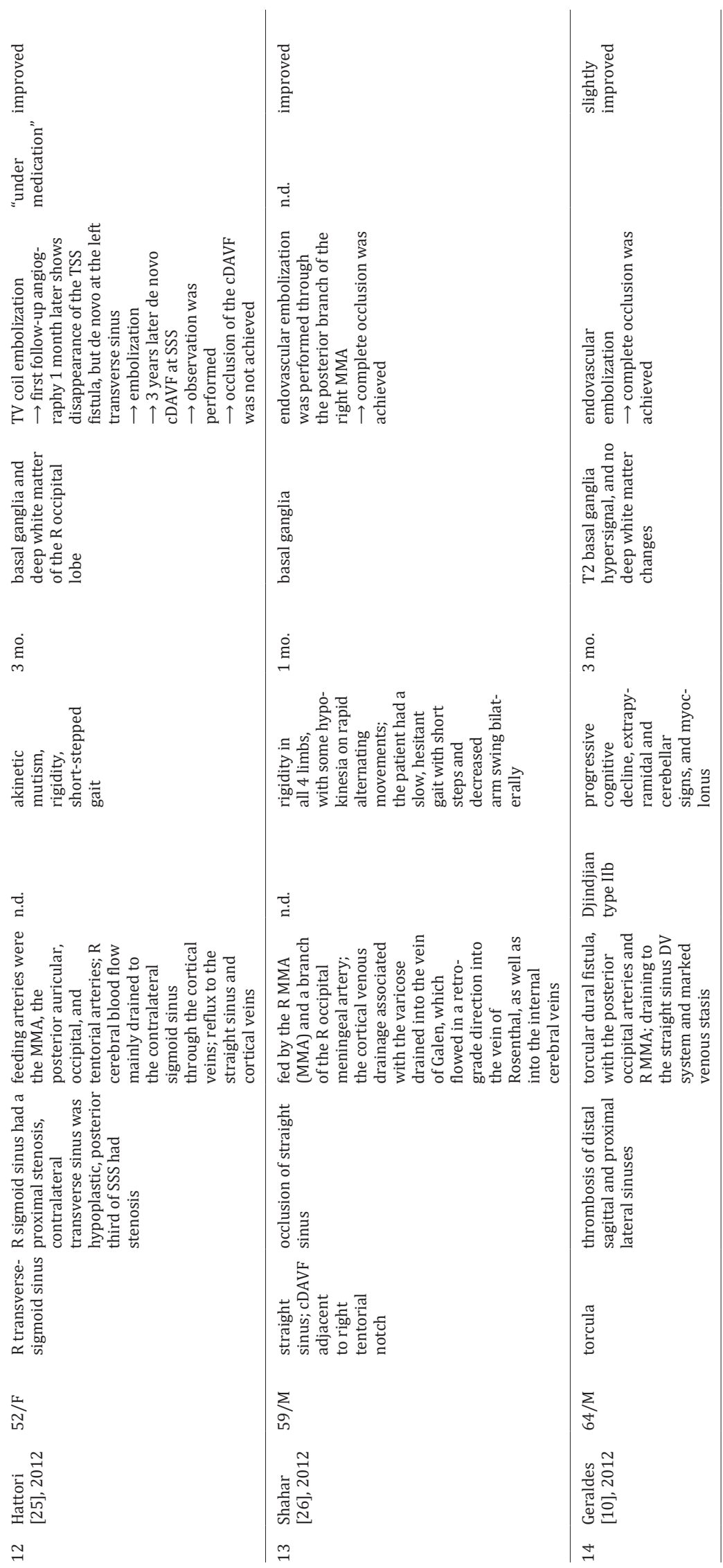

\section{Karger's}


Cerebrovascular Diseases Extra
Cerebrovasc Dis Extra 2020;10:124-138

Velz et al.: cDAVF and Secondary Parkinson's Syndrome

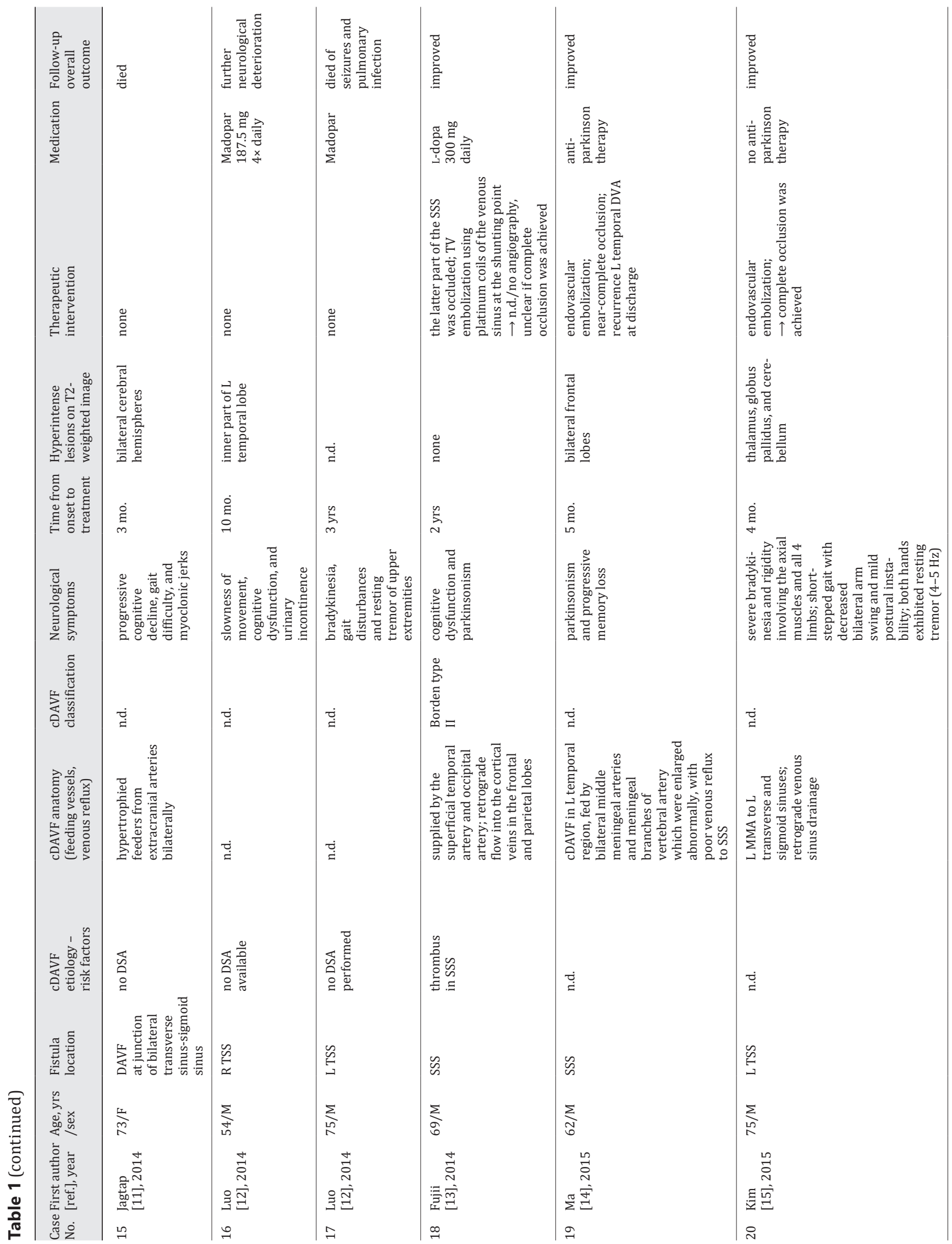



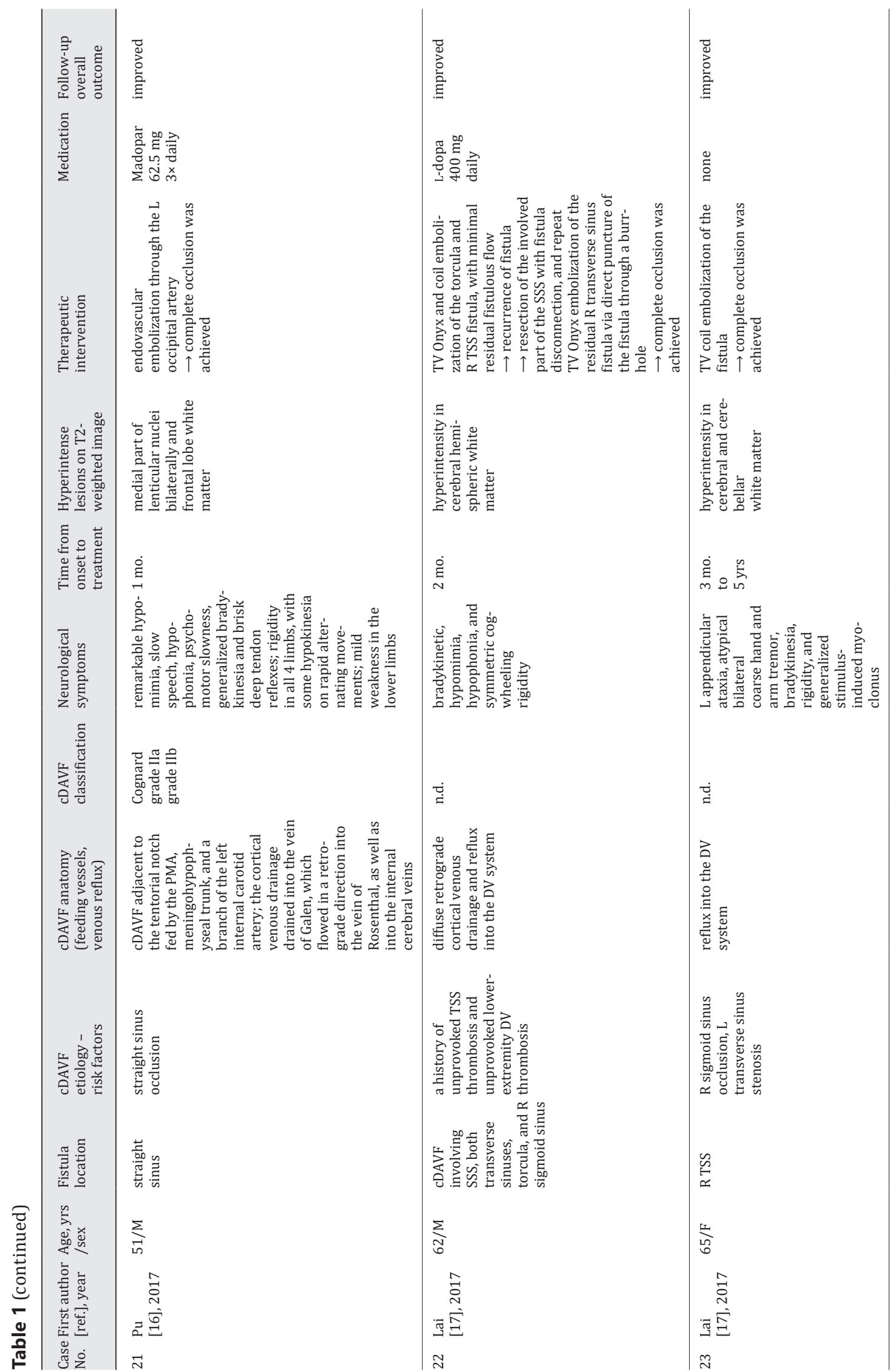

\section{Karger'}


Velz et al.: cDAVF and Secondary Parkinson's Syndrome

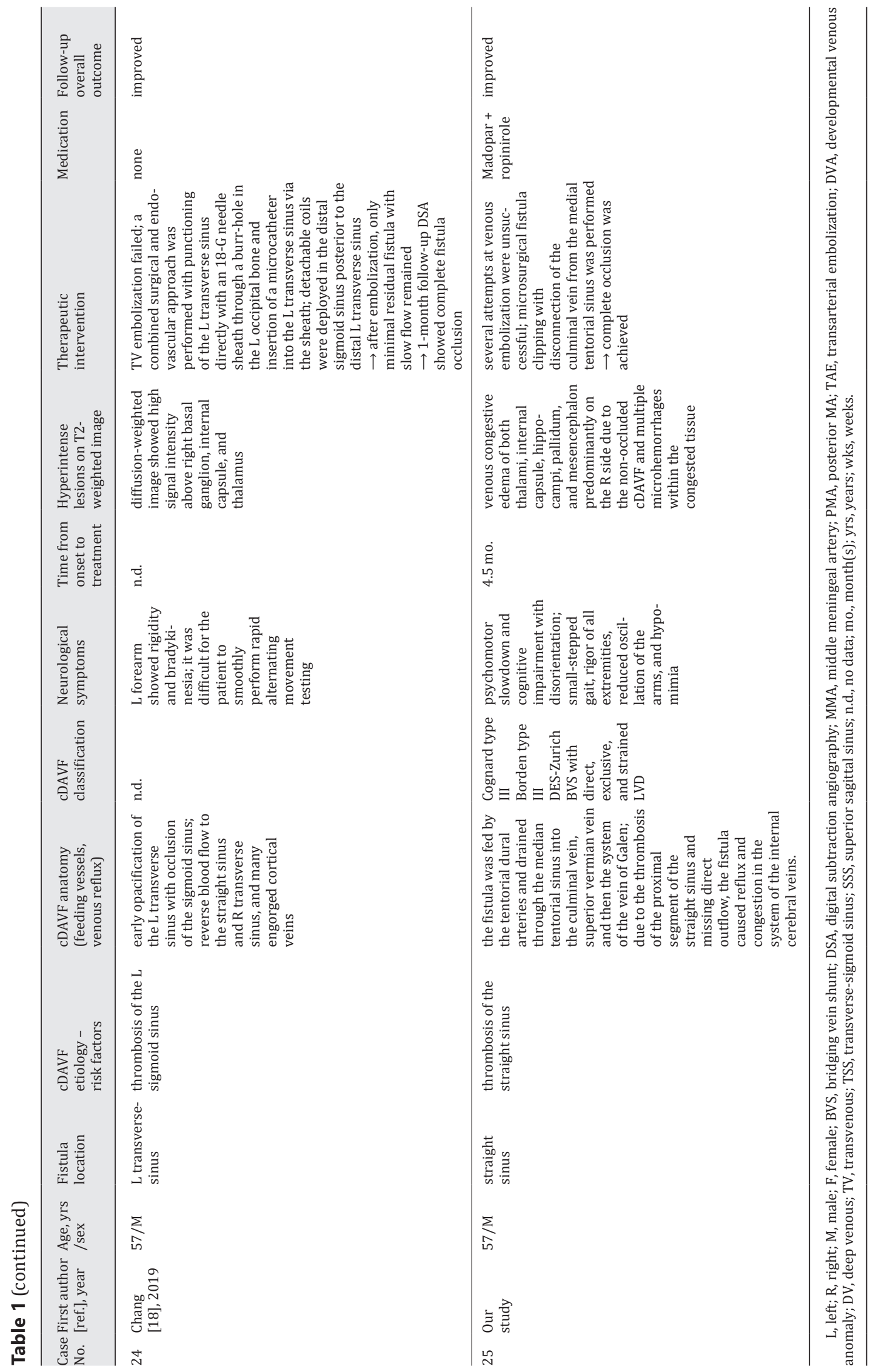

\section{Karger'}




\section{Cerebrovascular \\ Diseases Extra}

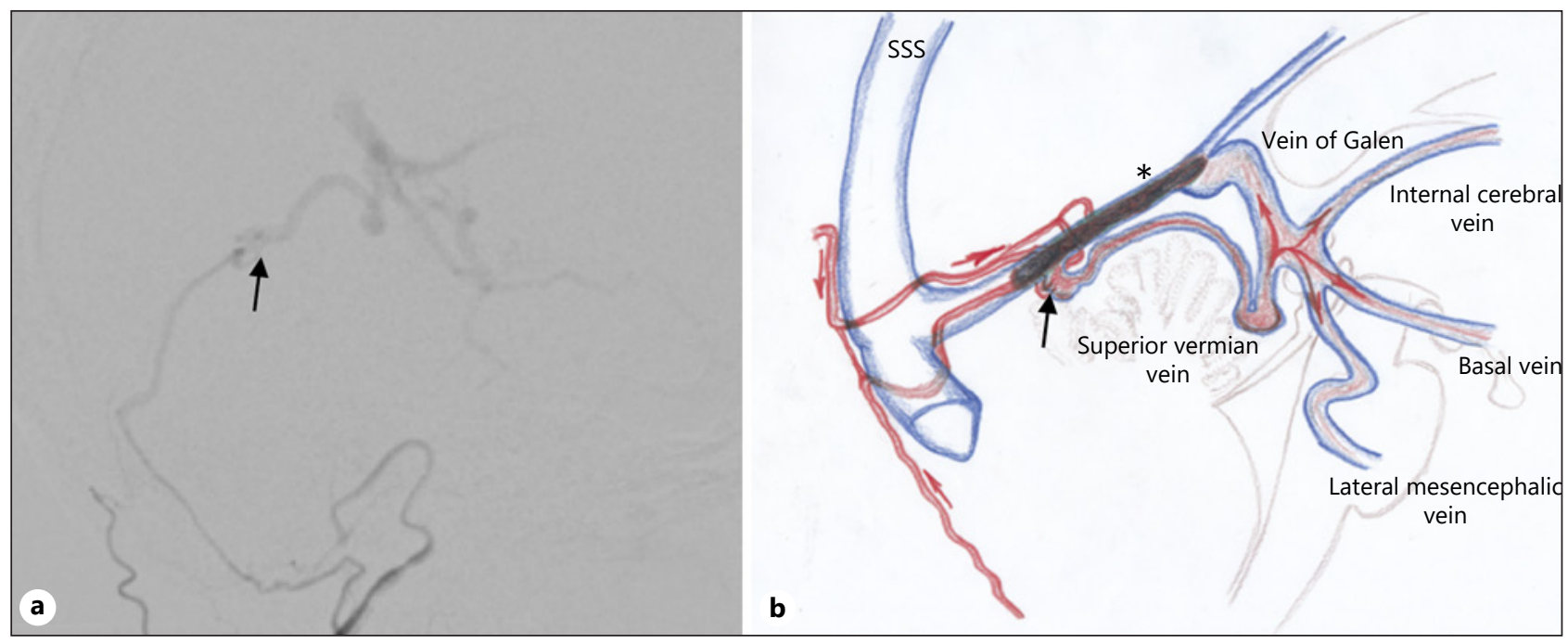

Fig. 1. a DSA reveals an arteriovenous shunt (arrow) at the level of the thrombosed part of the straight sinus. b Graphic illustration of the cDAVF. The fistula is fed by the tentorial dural arteries (red) and drains through the straight sinus into the median tentorial sinus, supraculminal vein, superior vermian vein, and then into the system of the vein of Galen. Due to the thrombosis (asterisk) of the proximal segment of the straight sinus, missing direct outflow, and arterialization of the system of the vein of Galen with consecutive flow reversion, the fistula causes severe reflux and congestion in the system of the internal cerebral veins. The cDAVF is classified as Cognard type III, Borden type III, and DES-Zurich bridging vein shunt type with direct, exclusive, and strained LVD. SSS, superior sagittal sinus.

icant medical history. After unspecific cranial computed tomography (CT) and cerebrospinal fluid examination, cranial magnetic resonance imaging (MRI) revealed partial thrombosis of the straight sinus with diffuse edema of both thalami, the internal capsule, the hippocampi, the pallidum, and the base of the mesencephalon (right > left) as well as multiple microbleeds in the basal ganglia and dilated deep cerebral veins.

Digital subtraction angiography (DSA) was performed, which showed a typical DAVF at the level of the thrombosed part of the straight sinus. The fistula was fed by the tentorial dural arteries and drained through the straight sinus into the median tentorial sinus, supraculminal vein, superior vermian vein, and the system of the vein of Galen. Due to the thrombosis of the proximal segment of the straight sinus, missing direct outflow, and arterialization of the system of the vein of Galen with consecutive flow reversion, the fistula caused severe reflux and congestion in the system of the internal cerebral veins (Fig. 1a, b). It was thus classified as Cognard type III, Borden type III, and DES-Zurich BVS type with direct, exclusive, and strained LVD (Fig. 1).

At an external hospital, several attempts at venous embolization of the fistula were not successful. Transarterial embolization was not performed due to high-grade outlet stenosis of the left vertebral artery. A transarterial embolization attempt via the transosseous-supplied posterior meningeal artery did not seem promising and was therefore not attempted. The patient was referred to our department 6 weeks after initial presentation.

At the time of admission, the patient showed severe neurological impairment and was not able to take care of himself, requiring considerable assistance and frequent medical care (Karnofsky performance score [KPS] of 30).

Repeated MRI and magnetic resonance angiography (MRA) revealed a massive progression of the venous congestive edema of both thalami, the internal capsule, the hippo- 


\section{Cerebrovascular \\ Diseases Extra}

\begin{tabular}{l|l}
\hline Cerebrovasc Dis Extra 2020;10:124-138 \\
\hline DOI: 10.1159/000510597 & $\begin{array}{l}\text { @ 2020 The Author(s). Published by S. Karger AG, Basel } \\
\text { www.karger.com/cee }\end{array}$ \\
\hline
\end{tabular}
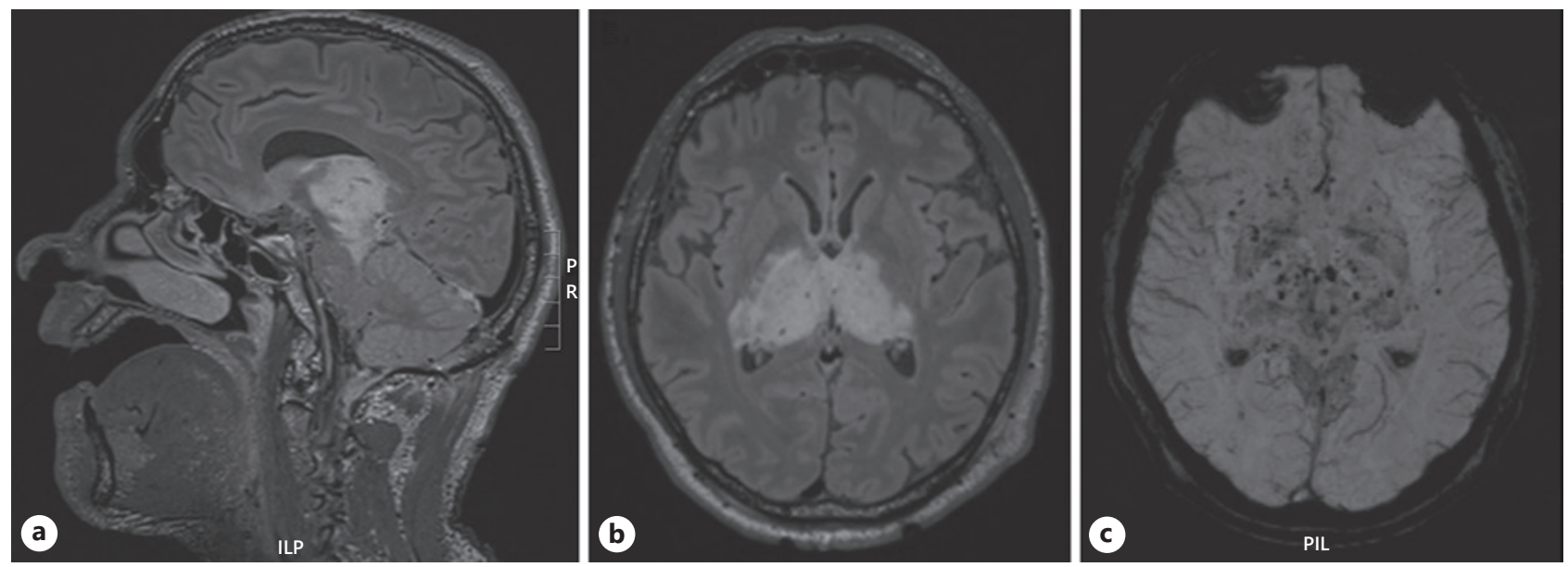

Fig. 2. a, b Sagittal and axial section of FLAIR-weighted images show a massive progression of the venous congestive edema of both thalami, internal capsule, pallidum and mesencephalon due to the nonoccluded cDAVF. c SWI-weighted images show multiple microhemorrhages within the congested tissue.

campi, the pallidum, and the mesencephalon, predominantly on the right side, due to the nonoccluded cDAVF (Fig. 2a, b), and also multiple microhemorrhages within the congested tissue (Fig. 2c).

A suboccipital paramedian right-sided mini-craniotomy was performed. Microsurgical opening of the cisterna magna was performed to facilitate supracerebellar infratentorial access and to identify the fistula draining into the supraculminal and superior vermian veins. Verification of its arterialization was done by fluorescein angiography. The culminal vein was disconnected from the medial tentorial sinus by bipolar electrocoagulation, achieving an immediate fistula occlusion. Verification of the successful disconnection was also done by fluorescein angiography. Postoperative MRA and DSA showed complete obliteration of the cDAVF (Fig. 3b, d). During hospitalization, a detailed examination of thrombophilia was performed due to the unexplained thrombosis of the straight sinus. There was no evidence of thrombophilia or oncological disease. At discharge, 12 days after surgery, the patient showed a significantly improved general condition. He was, however, not able to walk by himself and his cognitive impairment was still severe (a KPS of 60).

Carbidopa/levodopa was started based on the diagnosis of a secondary akinetic rigid Parkinson syndrome, and the neurological condition of the patient further improved. In the further course of the treatment, the dose was increased and ropinirole was added so that the patient was free of Parkinson symptoms when he left the rehabilitation clinic.

Three months later, the patient presented at our outpatient clinic in a significantly improved clinical condition (a KPS of 80-90). There were no signs of parkinsonism and his cognitive condition had improved. MRI showed complete regression of the bithalamic edema, internal capsule, hippocampi, pallidum, and mesencephalon (Fig. 4b, d), although the thrombosis of the straight sinus had remained unchanged.

Due to the complete regression of the venous stasis edema and the working diagnosis of a secondary Parkinson syndrome, dopaminergic medication was slowly tapered with no subsequent deterioration of the patient observed. 

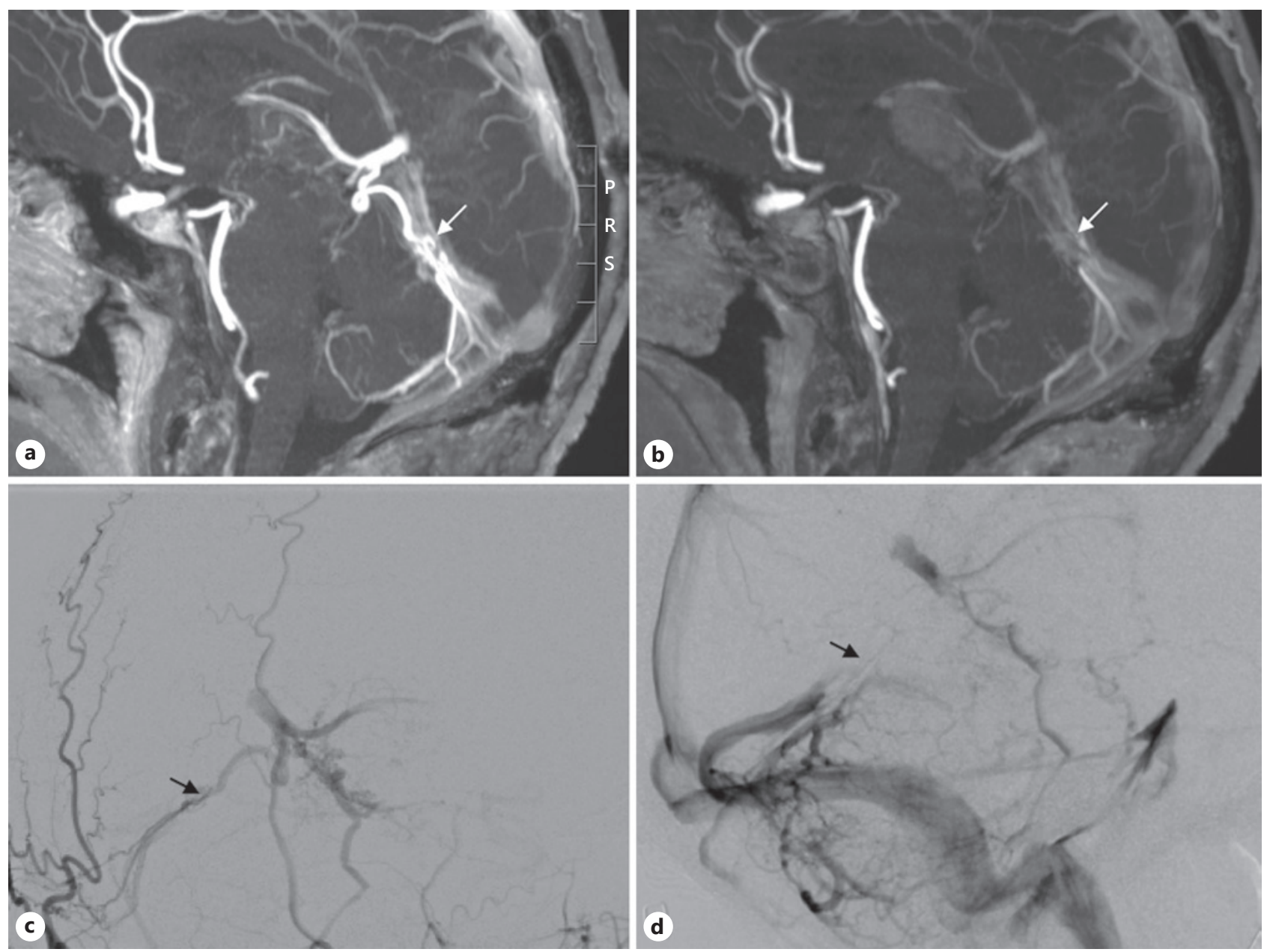

Fig. 3. Sagittal section of T1-weighted MRI TOF with gadolinium before (a) and after microsurgical clipping and complete cDAVF occlusion (b). DSA before (c) and after complete cDAVF occlusion (d).

\section{Discussion}

We present here the case of a 57-year-old male with a cDAVF of the straight sinus (Borden type III, Cognard type III, DES - Zurich BVS-type with direct, exclusive, and strained LVD) and worsening venous congestion of the Galenic system. He presented clinically with executive dysfunction, cognitive impairment, and disorientation. After complete microsurgical fistula occlusion, the bithalamic edema (with involvement of the internal capsule, hippocampi, and mesecencephalon) regressed. Concomitantly, parkinsonism disappeared and his functional impairment improved significantly.

Parkinsonism as a clinical manifestation of cDAVF is exceptionally rare and has so far only been reported in case reports and small case series [27]. We identified 24 cases described as having cDAVF and associated parkinsonism in the literature (Table 1). AV shunting into the transverse and sigmoid sinuses $(n=14 ; 58.3 \%)$ was most often described, followed by the superior sagittal sinus $(n=3 ; 12.5 \%)$, the torcular, straight sinus, and multiple sinus involvement (each $n=2 ; 8.3 \%)$, and anterior cranial fossa $(n=1 ; 4.2 \%)$. Retrograde filling of the straight sinus and/or impaired drainage of the deep internal venous system were 

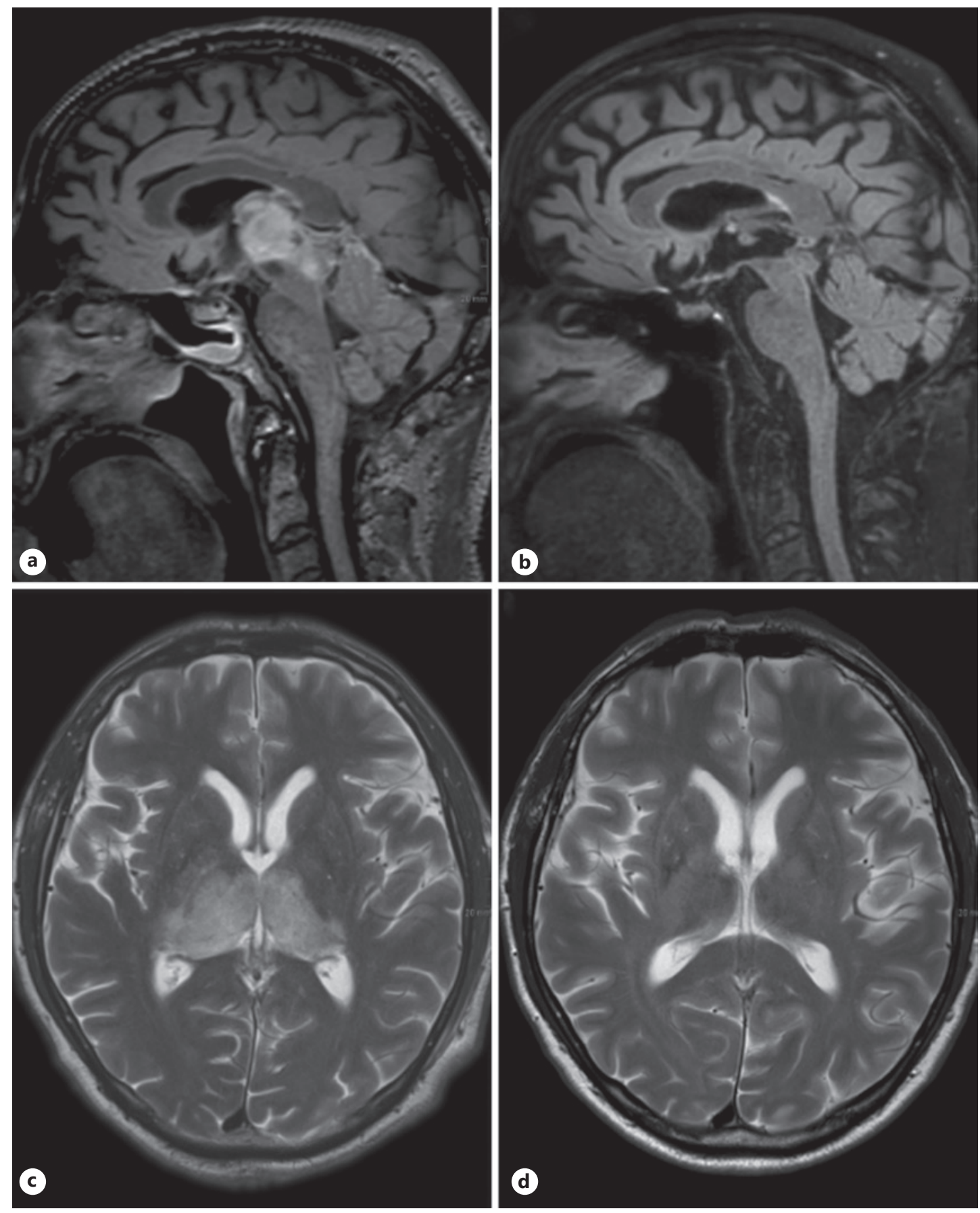

Fig. 4. Sagittal section of FLAIR (a, b) and axial section of T2-weighted (c, d) MRI. The 3-month follow-up MRI displays complete regression of the venous congestion of the Galenic system $(\mathbf{b}, \mathbf{d})$ compared to preoperatively (a, b).

described in 16 patients (66.6\%). Limited data or no data on the venous reflux were provided for 8 patients $(33.3 \%)$.

Lee et al. [20] were the first to propose the theory of basal ganglia dysfunction due to impaired drainage of the deep internal veins in patients with cDAVF and parkinsonism. They 
described a 60-year-old patient with cDAVF of the left sigmoid sinus with retrograde filling of the deep venous system, and superior sagittal sinus with prominent cortical venous reflux [20]. The patient showed reversible parkinsonism after embolization, which was well correlated with an increase in the basal ganglia-cerebellar perfusion ratio on SPECT, suggesting that a perfusion defect was responsible for the pathogenesis of the parkinsonism [20].

Kim et al. [15] reported a patient with decreased ${ }^{18} \mathrm{~F}$-FP-CIT uptake in cDAVF-associated parkinsonism. They suggested that hemodynamic impairment could cause parkinsonism via an accentuation of the underlying dopamine deficiency in subjects with preclinical-stage parkinsonism. The parkinsonism in their patient improved following the endovascular occlusion of the cDAVF without antiparkinson drug therapy [15].

In our case, both the presynaptic dopaminergic neurons in the mesencephalon and the postsynaptic neurons in the striatum, essential to mediate the treatment effect of dopaminergic medication, were affected by the edema (Fig. 2a, b). In addition, given the fact that carbidopa/levodopa was started during the process of neurological improvement, it remains unclear if the first signs of improvement during rehabilitation were related to this dopaminergic medication or to surgery. We believe it is more likely that the microsurgical disconnection of the cDAVF was primarily responsible for the clinical improvement during rehabilitation. The medication could therefore be tapered later without symptom recurrence. In the literature review, it was found that dopaminergic medication did not lead to improvement in 4 patients $[13,17,20,24]$ and that a transient response was reported in 3 patients $[12,16]$.

Treatment of patients with cDAVF and LVD in the deep venous system with secondary parkinsonism and/or cognitive impairment is challenging, as a remission of symptoms has been described in only a few cases in which complete fistula occlusion was achieved. The literature review illustrates the severe natural history of cDAVF; 2 patients died (no intervention), and 3 patients showed severe neurological deterioration (1 had no intervention and 2 attempted unsuccessful embolization). These numbers might even be underestimated due to limited data on follow-up in the cases described and the known general bias against reporting poor results $[9,11,12,24]$.

Our aim is to highlight the importance of rapid diagnosis and complete occlusion of the cDAVF to reverse parkinsonism and prevent further neurological deterioration, including death. Interestingly, complete cDAVF occlusion was achieved in only 8 of 24 (33.3\%) and incomplete occlusion in 5 patients $(20.8 \%)$. In 3 patients (12.5\%), no intervention was made; insufficient data were available for 8/24 (33.3\%) (Table 1). In all the reported cases, endovascular embolization was the 1 st treatment choice. In 3 cases, no intervention was performed; in 1 case, no information was available about the treatment used. Surprisingly, no cDAVF in this literature review was treated by microsurgical cDAVF disconnection, despite complete occlusion being achieved in only 8/24 (33.3\%) patients. Endovascular embolization was repeated in a few cases when the first attempt was unsuccessful (Table 1). In 3 cases, a combined endovascular and surgical approach was performed to gain access to the cDAVF for endovascular treatment, but microsurgical clipping/disconnection of the cDAVF was not performed in these cases (Table 1) [17, 18, 23].

Even in cases where only incomplete cDAVF occlusion was achieved through endovascular embolization, a "watch-and-wait-strategy" was the preferred strategy, exposing the patient to persistent LVD and the risk of clinical deterioration.

The detailed understanding of the angioarchitecture of the cDAVF is essential to determine the treatment strategy. It is based on the precise identification of the AV shunt localization (BVS, DSS, or EVS) as well as on the understanding of the LVD. A detailed review of the angioarchitecture of cDAVF is beyond the scope of this report and we refer the interested reader to the description by Baltsavias and Valavanis $[1-3,6]$. To achieve complete cDAVF occlusion, a multidisciplinary discussion of the endovascular and microsurgical options is recom-

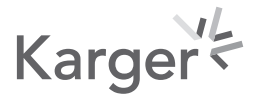


mended. Although we favor endovascular surgery as the first-line treatment, microsurgery should be considered in cases of persistence of an AV shunt. The surgical approach is often straightforward and can be easily performed by an experienced vascular neurosurgeon. Despite a preoperatively detailed understanding of the angioarchitecture of the cDAVF, intraoperative identification of the AV shunt might pose a challenge; confirmation of the AV shunt as well as the successful disconnection should therefore be verified by intraoperative fluorescein angiography [28].

Patients with cDAVF diagnosed as BVS, the majority of which are Borden type III, are excellent candidates for primary microsurgical cDAVF occlusion [29]. The surgical approach is straightforward and can be done through a simple mini-craniotomy by simply disconnecting the draining vein, regardless of the presence or absence of venous strain [29].

\section{Statement of Ethics}

The subject gave his written informed consent to publish this case. In addition, the institutional review board approved the use of registry data for clinical research, registered under the case No. KEK-ZH 2012-0244.

\section{Conflict of Interest Statement}

None of the authors report a conflict of interest related to this report.

\section{Funding Sources}

This research did not receive any specific grant from funding agencies in the public, commercial, or not-for-profit sectors.

\section{Author Contributions}

J.V., Z.K., and L.R.: conception and design; acquisition, analysis, and interpretation of data; drafting the article. F.B. and H.R.: acquisition, analysis, and interpretation of data.

\section{References}

1 Baltsavias G, Parthasarathi V, Aydin E, Al Schameri RA, Roth P, Valavanis A. Cranial dural arteriovenous shunts. Part 1. Anatomy and embryology of the bridging and emissary veins. Neurosurg Rev. 2015 Apr;38(2):253-63.

2 Baltsavias G, Kumar R, Avinash KM, Valavanis A. Cranial dural arteriovenous shunts. Part 2. The shunts of the bridging veins and leptomeningeal venous drainage. Neurosurg Rev. 2015 Apr;38(2):265-71.

3 Baltsavias G, Roth P, Valavanis A. Cranial dural arteriovenous shunts. Part 3. Classification based on the leptomeningeal venous drainage. Neurosurg Rev. 2015 Apr;38(2):273-81.

4 Borden JA, Wu JK, Shucart WA. A proposed classification for spinal and cranial dural arteriovenous fistulous malformations and implications for treatment. J Neurosurg. 1995 Feb;82(2):166-79.

5 Gerlach R, Boehm-Weigert M, Berkefeld J, Duis J, Raabe A, Seifert V, et al. Thrombophilic risk factors in patients with cranial and spinal dural arteriovenous fistulae. Neurosurgery. 2008 Oct;63(4):693-8.

6 Baltsavias G, Spiessberger A, Hothorn T, Valavanis A. Cranial dural arteriovenous shunts. Part 4. Clinical presentation of the shunts with leptomeningeal venous drainage. Neurosurg Rev. 2015 Apr;38(2):283-91.

7 Cognard C, Gobin YP, Pierot L, Bailly AL, Houdart E, Casasco A, et al. Cerebral dural arteriovenous fistulas: clinical and angiographic correlation with a revised classification of venous drainage. Radiology. 1995 Mar; 194(3):671-80. 


\section{Cerebrovascular Diseases Extra}

\begin{tabular}{l|l}
\hline Cerebrovasc Dis Extra 2020;10:124-138 \\
\hline DOI: 10.1159/000510597 & $\begin{array}{l}\text { @ 2020 The Author(s). Published by S. Karger AG, Basel } \\
\text { www.karger.com/cee }\end{array}$ \\
\hline
\end{tabular}

Velz et al.: cDAVF and Secondary Parkinson's Syndrome

8 Okuizumi K, Watanabe K, Yamazaki M, Koike T, Onishi Y. A case of dural arteriovenous malformation associated with progressive dementia showing marked improvement with endovascular treatment. Rinsho Shinkeigaku. 1998 Feb;38(2):112-7. Japanese.

9 Matsuda S, Waragai M, Shinotoh H, Takahashi N, Takagi K, Hattori T. Intracranial dural arteriovenous fistula (DAVF) presenting progressive dementia and parkinsonism. J Neurol Sci. 1999 May;165(1):43-7.

10 Geraldes R, Albuquerque L, Ferro JM, Sousa R, Sequeira P, Campos J. Rapidly progressive cognitive impairment, ataxia, and myoclonus: an unusual presentation of a dural arteriovenous fistula. J Stroke Cerebrovasc Dis. 2012 Oct;21(7):619.e3-5.

11 Jagtap SA, Nair SS, Jain N, Nair MD. Rapidly progressive dementia, parkinsonism and myoclonus: an unusual presentation of dural arteriovenous fistula. Neurol India. 2014 Jan-Feb;62(1):107-10.

12 Luo Y, Qi J, Cen Z, Hu H, Jiang B, Luo W. Two cases of dural arteriovenous fistula presenting with parkinsonism and progressive cognitive dysfunction. J Neurol Sci. 2014 Aug;343(1-2):211-4.

13 Fujii H, Nagano Y, Hosomi N, Matsumoto M. Dural arteriovenous fistula presenting with progressive dementia and parkinsonism. BMJ Case Rep. 2014 Jun;2014:bcr2014203921.

14 Ma C, Lu Q, Shi W, Su Z, Zhao Y, Li C, et al. Diagnosis and treatment of a dural arteriovenous fistula presenting with progressive parkinsonism and dementia: A case report and literature review. Exp Ther Med. 2015 Feb; 9(2):523-6.

15 Kim HR, Lee J, Kim YK, Park H, Kim HJ, Son YJ, et al. Dural Arteriovenous Fistula-Associated Reversible Parkinsonism with Presynaptic Dopaminergic Loss. J Mov Disord. 2015;8(3):141-3.

$16 \mathrm{Pu}$ J, Si X, Ye R, Zhang B. Straight sinus dural arteriovenous fistula presenting with reversible parkinsonism: A case report and literature review. Medicine (Baltimore). 2017;96:49(e9005).

17 Lai J, Heran MK, Stoessl AJ, Gooderham PA. Reversible Parkinsonism and Rapidly Progressive Dementia Due to Dural Arteriovenous Fistula: Case Series and Literature Review. Mov Disord Clin Pract (Hoboken). 2017 Mar;4(4):607-11.

18 Chang CW, Hung HC, Tsai JI, Lee PC, Hung SC. Dural Arteriovenous Fistula With Sinus Thrombosis and Venous Reflux Presenting as Parkinsonism: A Case Report. Neurologist. 2019 Jul;24(4):132-5.

19 Hamada Y, Yamakawa Y, Fukui M. A case of dural arteriovenous fistula in the anterior cranial fossa presenting frozen gait. Jpn J Neurosurg. 2003;12(12):798-802.

20 Lee PH, Lee JS, Shin DH, Kim BM, Huh K. Parkinsonism as an initial manifestation of dural arteriovenous fistula. Eur J Neurol. 2005 May;12(5):403-6.

21 Kajitani M, Yagura H, Kawahara M, Hirano M, Ueno S, Fujimoto K, et al. Treatable fluctuating Parkinsonism and dementia in a patient with a dural arteriovenous fistula. Mov Disord. 2007 Feb;22(3):437-9.

22 Miura S, Noda K, Shiramizu N, Muraoka N, Hirohata M, Ayabe M, et al. Parkinsonism and ataxia associated with an intracranial dural arteriovenous fistula presenting with hyperintense basal ganglia in T1-weighted MRI. J Clin Neurosci. 2009 Feb;16(2):341-3.

23 Nogueira RG, Baccin CE, Rabinov JD, Pryor JC, Buonanno FS, Hirsch JA. Reversible parkinsonism after treatment of dural arteriovenous fistula. J Neuroimaging. 2009 Apr;19(2):183-4.

24 Netravathi M, Pal PK, Bharath RD, Ravishankar S. Intracranial dural arteriovenous fistula presenting as parkinsonism and cognitive dysfunction [Internet]. J Clin Neurosci. 2011 Jan;18(1):138-40.

25 Hattori T, Takeuchi T, Kabeya R, Ando K, Tosaki F. Transverse-sigmoid sinus dural arteriovenous fistula presenting with parkinsonism. Neurol Med Chir (Tokyo). 2013;53(4):224-7.

26 Shahar T, Gadoth A, Nossek E, Giladi N, Ram Z, Maimon S. Reversible freezing of gait caused by dural arteriovenous fistula and congestion of the globus pallidus. Mov Disord. 2012 Nov;27(13):1690-3.

27 Brito A, Tsang AC, Hilditch C, Nicholson P, Krings T, Brinjikji W. Intracranial Dural Arteriovenous Fistula as a Reversible Cause of Dementia: Case Series and Literature Review. World Neurosurg. 2019 Jan;121:e543-53.

28 Esposito G, Dias S, Burkhardt JK, Bozinov O, Regli L. Role of indocyanine green videoangiography in identification of donor and recipient arteries in cerebral bypass surgery. Acta Neurochir Suppl (Wien). 2018;129: 85-9.

29 Baltsavias G, Valavanis A, Regli L. Cranial dural arteriovenous shunts: selection of the ideal lesion for surgical occlusion according to the classification system. Acta Neurochir (Wien). 2019 Sep;161(9):1775-81. 